\title{
Modeling of propagation of optical signals in gradient index media based on fractional Fourier transform
}

\author{
Zubtsov R.O., \\ Samara State Aerospace University \\ Kirilenko M.S. \\ Samara State Aerospace University, \\ Image Processing Systems Institute, Russian Academy of Sciences
}

\begin{abstract}
This research has simulated the propagation of the light beams through the quadratic index media. Five methods of simulation were considered and the propagating beams corresponding to different input signals such as Airy-Gaussian beams, rectangular function pulses, triangular function pulses, cosine function signals and finite eigenfunctions were demonstrated.
\end{abstract}

Keywords: gradient index media, fractional Fourier transform, Airy-Gaussian beams, Hermite-Gaussian modes, eigenfunctions

Citation: Zubtsov RO, Kirilenko MS. Modeling of propagation of optical signals in gradient index media based on fractional Fourier transform. Proceedings of Information Technology and Nanotechnology (ITNT-2015), CEUR Workshop Proceedings, 2015; 1490: 105-111. DOI: 10.18287/16130073-2015-1490-105-111

\section{Introduction}

Fractional Fourier transform (FrFT) is a set of linear transformations that generalize Fourier transform. Fourier transform is generally interpreted as a convention of the time domain of the signal to its frequency domain.

The canonical FrFT was considered [1] as the Fourier transform of $\alpha$-order, where $\alpha$ is the real value. We can likewise define the FrFT as the operation of the frequencytime distribution (Wigner distribution function) rotation at a certain angle [2].

Originally, FrFT was used in quantum mechanics; however, recently it has increasingly become a focus of opticists. As a result, extensive research involving its properties, optical realization and potentiality opportunities in optic applications has been performed. Thus, currently FrFT is actively used in optical image processing [3]. Moreover, the fractioning of some transformation provides a new degree of freedom (fraction order) that can be used for a more complete description of the object (signal) or as an additional encoding parameter.

FrFT is used in differential equation solving, in quantum mechanics and quantum optics, in optical theory of diffraction, in optical system and optical signal processing 
descriptions including the application of frequency filters, time filtration and multiplexing, as well as in pattern recognition, in wavelet-transformations, in operations with chirp-functions, in encryption, for neural network creation and other applications. A more detailed review of FrFT can be found in the paper [3] produced by $\mathrm{T}$. Alieva et al.

The modular lens system and the system of several spherical and/or cylindrical lenses are among the methods of FrFT optical realization [4-8]. Some of these systems (especially cylindrical lenses) are used for astigmatic transformation in order to form vortex beams $[5,8-10]$.

One of application of FrFT is the description of laser beam propagation in gradient index media $[11,12]$.

In this work, we use one-dimensional FrFT to model optical signal propagation in optical waveguides with parabolic dependence of the refractive index. The eigenfunctions of the transforms are Hermite-Gaussian modes [1].

During the modelling process special attention is given to Airy-Gaussian beams, which carry finite power and keep the properties of non-diffracted propagation in a partial area. They can be experimentally realized with a particularly good approximation [13-16].

\section{General theory}

The light beam propagation through the ABCD-system in one-dimensional cases is described by the Huygens integral:

$U_{2}\left(x_{2}\right)=\sqrt{\frac{k}{i 2 \pi B}} \int_{-\infty}^{+\infty} U_{1}\left(x_{1}\right) \exp \left[\frac{i k}{2 B}\left(A x_{1}^{2}-2 x_{1} x_{2}+D x_{2}^{2}\right)\right] d x_{1}$.

where $k=2 \pi / \lambda, U_{1}\left(x_{1}\right)$ is input field, $U_{2}\left(x_{2}\right)$ is output field.

In gradient index media with the refraction index $n=n_{0}\left(1-x^{2} / 2 a^{2}\right)$, the matrix of ABCD-system is (beam propagation from $z_{1}=0$ to $z_{2}=z$ ) $[13,14,17,18]$ :

$$
\left[\begin{array}{ll}
A & B \\
C & D
\end{array}\right]=\left[\begin{array}{cc}
\cos (z / a) & a \sin (z / a) \\
-\sin (z / a) / a & \cos (z / a)
\end{array}\right] .
$$

With this type of matrix the integral (1) turns into FrFT.

The complete set of FrFT eigenfunctions is the following set of Hermite-Gaussian functions:

$$
\begin{aligned}
& \mathfrak{J}^{\alpha}\left[\psi_{n}(x)\right]=e^{-i \alpha n \pi / 2} \psi_{n}(x), \\
& \psi_{n}(x)=\frac{2^{1 / 4}}{\sqrt{2^{n} n !}} H_{n}(\sqrt{2 \pi} x) e^{-\pi x^{2}},
\end{aligned}
$$

where $H_{n}(x)$ - a Hermite polynomial of order $n$ :

$$
H_{n}(x)=(-1)^{n} e^{x^{2}} \frac{d}{d x^{n}} e^{-x^{2}}
$$

If the FrFT has finite integration limits (in other words the input beam is limited), its eigenfunctions are somewhat different from the Hermite-Gaussian modes [19]. 
The propagation of a light beam through the gradient index media was modeled.

The Airy-Gaussian beams are:

$$
\begin{aligned}
& U_{1}\left(x_{1} ; \kappa_{1}, \delta_{1}, S_{1}, q_{1}\right)= \\
& =A i\left(\frac{x_{1}+\delta_{1}}{\kappa_{1}}\right) \exp \left[i S_{1}\left(\frac{x_{1}+\delta_{1}}{\kappa_{1}}\right)+i \frac{S_{1}^{2}}{3}+\frac{i k x_{1}^{2}}{2 q_{1}}\right], \\
& \kappa_{1}, \delta_{1}, S_{1}, q_{1} \in C .
\end{aligned}
$$

Furthermore, the cosine distribution function is:

$$
f(x)=A \cos (\omega x+\varphi) \text {, }
$$

$A, \omega, \varphi \in R$.

In addition, the rectangular function, triangle function and eigenfunctions have been simulated.

\section{Simulation}

Method 1: direct numerical calculation of FrFT by definition.

The integral (1) is rarely solved analytically, so we use numerical computations. If typical methods are use, the numerical calculations of quadratic exponents require a very large number of sampling points because of rapid oscillations in the kernel. The problem is especially pronounced if $\alpha$ is close to 0 or \pm 2 . We assume that functions and their Fourier transformations are limited (they are not equal to zero in finite intervals) and this difficulty can be avoided. If $0.5 \leq \alpha \leq 1.5$ or $2.5 \leq \alpha \leq 3.5$, we can directly calculate the integral. If $-0.5<\alpha<0.5$ or $1.5<\alpha<2.5$, we can use the property of additivity: $\mathfrak{I}^{\alpha}=\mathfrak{I}^{1} \mathfrak{I}^{\alpha-1}$, where in the transformation of $\alpha-1$ order may be found immediately.

For the integration we use Simpson's rule ( $n$ is even):

$$
\begin{aligned}
& \int_{a}^{b} f(x) d x \approx \frac{h}{3} \sum_{j=1}^{n / 2}\left(f\left(x_{2 j-2}\right)+4 f\left(x_{2 j-1}\right)+f\left(x_{2 j}\right)\right), \\
& x_{j}=a+j h, j=\overline{0, n-1}, h=(b-a) / n .
\end{aligned}
$$

Although this method of FrFT calculation can produce accurate results, it operates slowly and has a computational complexity $O\left(N^{2}\right)$ [20].

Method 2: Fast FrFT.

FrFT is a special case of the more general transform class sometimes known as linear canonical transformations or quadratic-phase transformations. The elements of this class can be decomposed to a sequence of simple operations such as chirpmultiplication, chirp-convolution, scaling and typical Fourier transform. There are two different decompositions demonstrated here, leading to different algorithms.

Method 3: the decomposition into chirp-multiplication, chirp-convolution and another single chirp-multiplication sequence.

In this approach, we assume that $-1 \leq \alpha \leq 1$. The transformation (1) can be written as:

$$
f_{\alpha}(x)=\exp \left[-\frac{i \pi}{\lambda a} x^{2} \tan \left(\frac{\varphi}{2}\right)\right] g^{\prime}(x),
$$




$$
\begin{aligned}
& g^{\prime}(x)=A_{\varphi} \int_{-\infty}^{+\infty} \exp \left[\frac{i \pi}{\lambda a} \beta\left(x-x^{\prime}\right)^{2}\right] g\left(x^{\prime}\right) d x^{\prime}, \\
& g(x)=\exp \left[-\frac{i \pi}{\lambda a} x^{2} \tan \left(\frac{\varphi}{2}\right)\right] f(x)
\end{aligned}
$$

where $g(x), g^{\prime}(x)$ represents an intermediate result, $\varphi=\frac{z}{a}, \beta=\csc \varphi, A_{\varphi}=\sqrt{\frac{\beta}{i \lambda a}}$.

Method 4: another decomposition involving the Whittaker-Shannon interpolation formula (sinc-interpolation). The defining expression of FrFT can be written as:

$$
\begin{aligned}
& \left\{\mathfrak{J}^{\alpha} f\right\}(x)=A_{\varphi} \exp \left(\frac{i \pi}{\lambda a} \alpha x^{2}\right) \times \\
& \times \int_{-\infty}^{+\infty} \exp \left(-\frac{i 2 \pi \beta}{\lambda a} x x^{\prime}\right) \exp \left(\frac{i \pi}{\lambda a} \alpha x^{\prime 2}\right) f\left(x^{\prime}\right) d x^{\prime} .
\end{aligned}
$$

The function $\exp \left(\frac{i \pi}{\lambda a} \alpha x^{\prime 2}\right) f\left(x^{\prime}\right)$ can be represented by the Shannon interpolation formula:

$$
\begin{aligned}
& \exp \left(i \pi \alpha x^{\prime 2}\right) f\left(x^{\prime}\right)=\sum_{-N}^{N} \exp \left[\frac{i \pi}{\lambda a} \alpha\left(\frac{n}{2 \Delta x}\right)^{2}\right] \times \\
& \times f\left(\frac{n}{2 \Delta x}\right) \operatorname{sinc}\left(2 \Delta x\left(x^{\prime}-\frac{n}{2 \Delta x}\right)\right) .
\end{aligned}
$$

We substitute (12) in (11), change sequence of integration and summation and use certain algebraic manipulations to obtain the following expression:

$$
\begin{aligned}
& \left\{\mathfrak{J}^{\alpha} f\right\}\left(\frac{m}{2 \Delta x}\right)=\frac{A_{\varphi}}{2 \Delta x} \exp \left[\frac{i \pi}{\lambda a}(\alpha-\beta)\left(\frac{m}{2 \Delta x}\right)^{2}\right] \times \\
& \times \sum_{-N}^{N} \exp \left[\frac{i \pi}{\lambda a} \beta\left(\frac{m-n}{2 \Delta x}\right)^{2}+\frac{i \pi}{\lambda a}(\alpha-\beta)\left(\frac{n}{2 \Delta x}\right)^{2}\right] f\left(\frac{n}{2 \Delta x}\right) .
\end{aligned}
$$

There are other methods, for example those described in [21], however they are not well suited for plotting images on a plane. The modeling results are demonstrated in Figures 1-6.

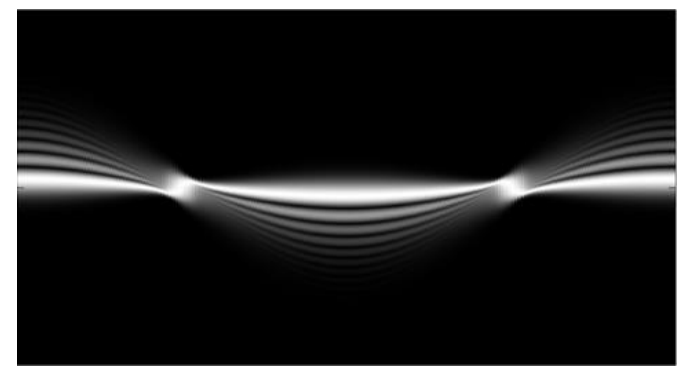

Fig. 1. - The propagation of Airy-Gaussian beams (version 1) 


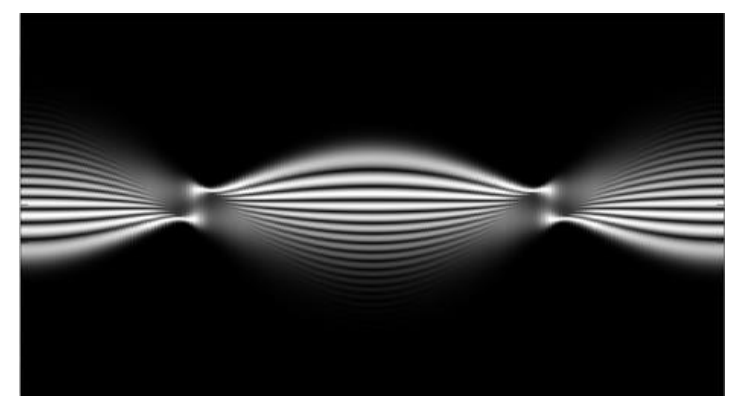

Fig. 2. - The propagation of Airy-Gaussian beams (version 2)

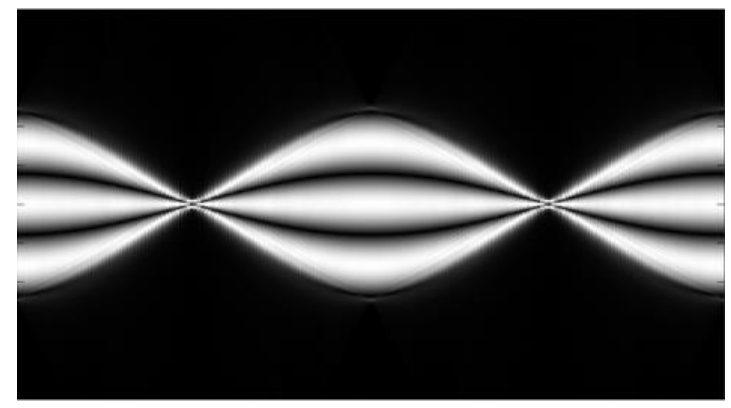

Fig. 3. - The propagation of cosine function signals

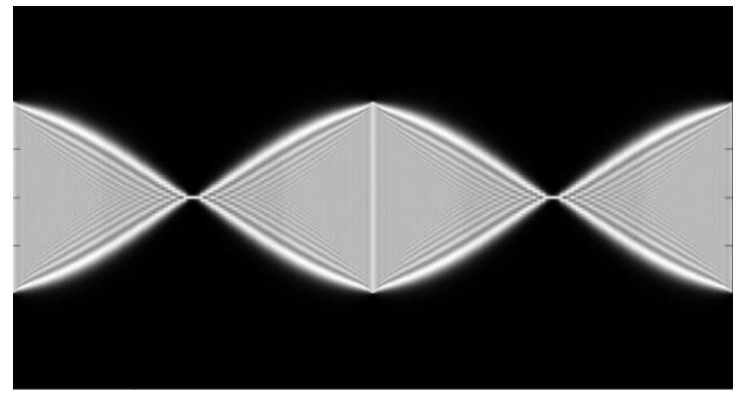

Fig. 4. - The propagation of rectangular pulses

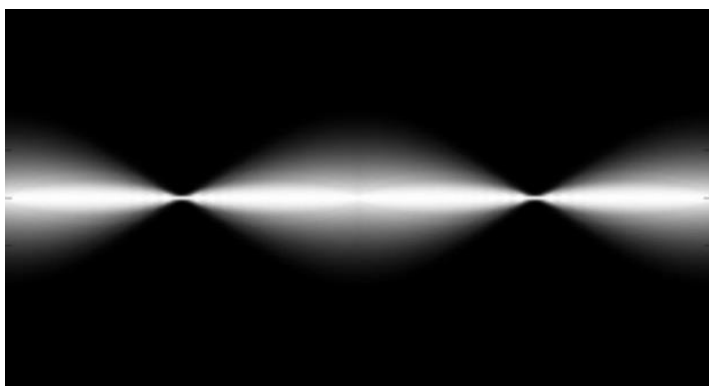

Fig. 5. - The propagation of triangular pulses 


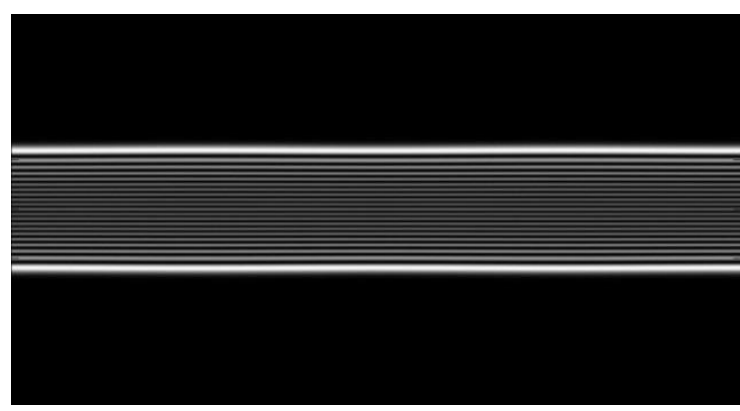

Fig. 6. - The propagation of eigenfunctions

\section{Acknowledgments}

This work was financially supported by the Russian Ministry of Education and Science and by the Russian Foundation for Basic Research (grant 13-07-00266).

\section{References}

1. Namias $\mathbf{V}$. The fractional Fourier transform and its application in quantum mechanics. Journal of the Institute of Mathematics and Its Applications, 1980; 25: 241-265.

2. Abet S, Sheridant JT. Generalization of the fractional Fourier transformation to an arbitrary linear lossless transformation: an operator approach. Journal of Physics A: Mathematical and General, 1994; 27: 4179-4187.

3. Alieva T, Bastiaans MJ, Calvo ML. Fractional transforms in optical information processing. EURASIP Journal on Advances in Signal Processing, 2005; 10: 1-22.

4. Dorsch RG, Lohmann AW. Fractional Fourier transform used for a lens-design problem. Applied Optics, 1995; 34(20): 4111-4112.

5. Cai LZ, Wang YQ. Optical implementation of scale invariant fractional Fourier transform of continuously variable orders with a two-lens system. Optics \& Laser Technology, 2002; 34: 249-252.

6. Malutin AA. Use of fractional Fourier-transformation in $\pi / 2$-converters of laser modes. Quantum Electronics, 2004; 2: 165-171.

7. Hahn J. Optical implementation of iterative fractional Fourier transform algorithm. Optics Express, 2006; 14(23): 11103-11112.

8. Khonina SN, Karpeev SV, Ustinov AV. Functional enhancement of mode astigmatic converters on the basis of application of diffractive optical elements. News of the Samara Science Center of the RAS, 2009; 11(5): 13-23.

9. Abramochkin E, Volostnikov V. Beams transformations and nontransformed beams. Optics Communications, 1991; 83: 123-135.

10. Beijersbergen MW, Allen L, van der Veen HELO, Woerdman JP. Astigmatic laser mode converters and transfer of orbital angular momentum. Optics Communications, 1993; 96: 123-132.

11. Ozaktas HM, Mendlovic D. Fourier transforms of fractional order and their optical interpretation. Optics Communications, 1993; 101: 163-169.

12. Mendlovic D, Ozaktas HM. Fractional Fourier transforms and their optical implementation: I. Journal of the Optical Society of America A, 1993; 10(9): 1875-1881.

13. Bandres MA, Gutiurrez-Vega JC. Airy-Gauss beams and their transformation by paraxial optical systems. Optics Express, 2007; 15(25): 16719-16728. 
14. Khonina SN, Volotovsky SG. Bounded 1D Airy beams: laser fan. Computer Optics, 2008; 32(2): 168-174. [in Russian]

15. Khonina SN, Skidanov RV, Moiseev OY. Airy laser beams generation by binary-coded diffractive optical elements for microparticles manipulation. Computer Optics, 2009; 33(2): 138-146. [in Russian]

16. Khonina SN. Specular and vortical Airy beams. Optics Communications, 2011; 284: 4263-4271

17. McMullin JN. The ABCD matrix in arbitrarily tapered quadratic-index waveguides. Applied Optics, 1986; 25: 2184.

18. Striletz AS, Khonina SN. Matching and investigation methods based on differential and integral operators of laser radiation propagation in a medium with small inhomogeneities. Computer Optics, 2008; 32(1): 33-38.

19. Kirilenko MS, Zubtsov RO, Khonina SN. Calculation of eigenfunctions of a bounded fractional Fourier transform. Computer Optics, 2015; 39(3): 332-338.

20. Ozaktas HM, Ankan O, Kutay A, Bozdaki G. Digital computation of the Fractional Fourier Transform. IEEE Transactions On Signal Processing, 1996; 44(9): 2141-2150.

21. Marinho FJ, Bernardo LM. Numerical calculation of fractional Fourier transforms with a single fast-Fourier-transform algorithm. Journal of the Optical Society of America A, 1998; 15(8): 2111-2116. 